\title{
Critical currents in the BEC-BCS crossover regime in an optical lattice
}

\author{
J. Tempere and J. T. Devreese \\ TFVS, Departement Fysica, Universiteit Antwerpen, Universiteitsplein 1, B-2610 Antwerpen, Belgium
}

(Received 1 August 2005; published 1 December 2005)

\begin{abstract}
Both the trapping geometry and the interatomic interaction strength of a dilute ultracold fermionic gas can be well controlled experimentally. Adapting the interaction strength between the fermionic atoms allows tuning the gas from a molecular condensate to a Bardeen-Cooper-Schrieffer superfluid. We adopt a functional integral point of view to investigate how the superfluid properties vary during this crossover. In particular, the critical superfluid velocity for flowing through an optical lattice is derived. The observation of undamped motion in an optical lattice constitutes a hallmark of superfluidity, also in the fermionic systems. We discuss our theoretical results for the critical velocity of a fermionic superfluid in comparison to the experimental results for a bosonic superfluid in an optical lattice.
\end{abstract}

DOI: 10.1103/PhysRevA.72.063601

PACS number(s): 03.75.Lm, 03.75.Ss

\section{INTRODUCTION}

The observation, ten years ago, of Bose-Einstein condensation (BEC) in a dilute gas of bosonic atoms has raised the challenge to achieve superfluidity in a gas of dilute fermionic atoms. The search for a superfluid Fermi gas has been helped to a large extent by the possibility to use Feshbach resonances to alter the interaction strength between the atoms [1]. When the interaction between fermionic atoms is attractive, a Bardeen-Cooper-Schrieffer (BCS) state can result below a critical temperature. Alternatively, the Feshbach resonance can be used to populate a bound state of a closed scattering channel, thus forming bosonic molecules that can Bose condense. In the crossover region near the Feshbach resonance, there is a strong coupling between the closed channel and the open scattering channel, so that the molecular state is strongly dressed [2]. As a result, the closed channel amplitude is typically small in the regions experimentalists are interested in [3].

The experimental realization of superfluidity in a Fermi gas in the molecular BEC regime came in 2003, when Zwierlein et al. [4] succeeded in creating a condensate of molecules formed out of fermionic atoms. The detection of superfluidity in the Bardeen-Cooper-Schrieffer (BCS) regime is much more subtle. In an initial experiment [5], the superfluid behavior was derived from the hydrodynamic nature of the expansion of the cloud, as compared to a ballistic expansion expected for a nonsuperfluid weakly interacting Fermi gas [6]. However, this did not constitute unambiguous proof, since the Fermi gas was in the strongly interacting regime. Subsequent experiments probed pairing by mapping the pair density onto a molecular condensate density [7] or by spectroscopically measuring the gap [8]. Although these experimental methods clearly demonstrate pairing, they do not unambiguously demonstrate superfluid behavior.

The very recent observation of a lattice of quantized vortices in resonant Fermi gases [9] constitutes the first clear demonstration of superfluidity in the BEC-BCS crossover regime. Observation of these vortices deep in the BCS regime may be difficult since the fermionic density penetrates in the core of the vortex in the BCS regime, leading to a loss of contrast in direct imaging [9-11]. Another possibility to demonstrate superconductivity is through the observation of the Josephson effect [12] in optical lattices. These optical lattices are periodic potentials formed by two counterpropagating laser beams, for example, in the $z$ direction

$$
V_{\text {opt }}(z)=s E_{R} \sin ^{2}(2 \pi z / \lambda),
$$

where $\lambda$ is the laser wavelength, $E_{R}=h^{2} /\left(2 m \lambda^{2}\right)$ is the recoil energy, and $s$ is the laser intensity expressed in units of the recoil energy. Typically, $s=0.1-20, \lambda=795 \mathrm{~nm}$. The atoms collect in the valleys of the optical lattice and form a "stack of pancakes." Typically, there are a few 100 "pancakes" with on the order of 1000 atoms each. When a superfluid is loaded in such an optical lattice, the system corresponds to an array of Josephson junctions. In such an array, the superfluid gas can propagate whereas the normal state gas is pinned. This has already been demonstrated for bosonic atoms [13], and has been predicted theoretically for fermionic atoms $[12,14]$. In this contribution, we derive and discuss the critical Josephson current for the flow of the superfluid component through an optical lattice. For this purpose, we base ourselves on the (one-channel) path-integral description as applied by Sá de Melo, Randeria, and Engelbrecht $[15,16]$ to the BEC-BCS crossover model of the high- $T_{c}$ superconductors.

\section{EFFECTIVE ACTION IN THE OPTICAL POTENTIAL}

The path-integral method has been applied before to describe vortices in a superfluid Fermi gas [11], and to describe the propagation of a superfluid Fermi gas in an optical potential [12]. When an optical lattice (1) is present along the $z$ direction, we can decouple the free motion in the $x, y$ plane from the tunneling motion in the $z$ direction. Taking this point of view, the system consists of a collection of twodimensional (2D) Fermi gases, indexed by a layer index $j$, and coupled to each other by the interlayer tunneling of atoms. The partition function of the system can then be represented as a functional integral over Grassmann variables $\bar{\psi}_{j, \sigma}, \psi_{j, \sigma}$ describing fermions, in layer $j$, with hyperfine spin $\sigma=\uparrow, \downarrow$, 


$$
\mathcal{Z}=\int \mathcal{D} \bar{\psi}_{j, \sigma}(r) \mathcal{D} \psi_{j, \sigma}(r) \exp \{-\mathcal{S} / \hbar\}
$$

with $r=\{x, y, \tau\}$ and

$$
\mathcal{S}=\sum_{j} \mathcal{S}_{j}+\sum_{j} \mathcal{S}_{j \rightarrow j+1}^{\text {tunnel }}
$$

The action functional is written as a sum of layer contributions $\mathcal{S}_{j}$ and tunneling contributions $\mathcal{S}_{j \rightarrow j+1}^{\text {tunnel }}$. The action functional for the fermions in layer $j$ is given by

$$
\begin{aligned}
\mathcal{S}_{j}= & \int_{0}^{\hbar \beta} d \tau \int d \mathbf{x} \sum_{\sigma} \bar{\psi}_{j, \sigma}(r)\left(\frac{\hbar \partial}{\partial \tau}-\frac{\hbar^{2} \nabla_{\mathbf{x}}^{2}}{2 m}-V_{j}-\mu\right) \psi_{j, \sigma}(r) \\
& +\int_{0}^{\hbar \beta} d \tau \int d \mathbf{x} g \bar{\psi}_{j, \uparrow}(r) \bar{\psi}_{j, \downarrow}(r) \psi_{j, \downarrow}(r) \psi_{j, \uparrow}(r)
\end{aligned}
$$

Here, $m$ is the mass of the fermions, $\mu$ is the chemical potential fixing the total number of fermions, and $\mathbf{x}=\{x, y\}$ is the (2D) position vector. Fermions in different hyperfine states interact through a contact potential with strength $g$. An external potential $V_{j}$ acts on each layer; this can be a parabolic potential in addition to the optical potential itself. The tunneling of atoms from one layer to another is described by

$$
\begin{aligned}
\mathcal{S}_{j \rightarrow j+1}^{\text {tunnel }}= & \int_{0}^{\hbar \beta} d \tau \int d \mathbf{x} t_{1} \sum_{\sigma}\left[\bar{\psi}_{j, \sigma}(r) \psi_{j+1, \sigma}(r)\right. \\
& \left.+\bar{\psi}_{j+1, \sigma}(r) \psi_{j, \sigma}(r)\right]
\end{aligned}
$$

where the tunneling energy $t_{1}$ bringing an atom from one well of the optical potential to the next was derived in Ref. [17]:

$$
t_{1}=s E_{R}\left[\frac{\pi^{2}}{4}-1\right] e^{-\sqrt{s}(\pi / 2)^{2}}
$$

For this particular decomposition of the action functional in intralayer contributions and tunneling contributions, we can perform the same analysis as described in Ref. [12]. A Hubbard-Stratonovich (HS) transformation gets rid of the four-operator term and allows us to introduce collective quantum fields $\left|\Delta_{j}\right|, \theta_{j}$ for the order parameter and the phase in each layer [18]. After making a saddle-point approximation for these fields, the integration over the Grassman variables can be performed. The final result for the effective action can again be written as the sum of contributions independent of $t_{1}$ and tunneling contributions

$$
\mathcal{S}_{\mathrm{eff}}=\sum_{j} \mathcal{S}_{\mathrm{eff}, j}+\sum_{j} \mathcal{S}_{\mathrm{eff}, j \rightarrow j+1}^{\text {tunnel }}
$$

The effective action for layer $j$ is

$$
\mathcal{S}_{\mathrm{eff}, j}=-\hbar \operatorname{tr}\left[\ln \left(\frac{-\mathrm{G}_{\mathrm{sp}}^{-1}}{\hbar}\right)\right]-\int_{0}^{\hbar \beta} d \tau \int d \mathbf{x} \frac{\left|\Delta_{j}\right|^{2}}{g},
$$

with

$$
-\mathrm{G}_{\mathrm{sp}}^{-1}=\sigma_{0}\left(\hbar \frac{\partial}{\partial \tau}\right)+\sigma_{3}\left(-\frac{\hbar^{2}}{2 m} \nabla_{\mathbf{x}}^{2}-V_{j}-\mu\right)-\sigma_{1}\left(\hbar\left|\Delta_{j}\right|\right),
$$

where $\sigma_{j}$ are the Pauli matrices. The tunneling contributions in the effective action (7) can be treated perturbatively. In that framework, the saddle-point values $\left|\Delta_{j}\right|$ can be extracted from the gap equation obtained by extremizing (8) in each layer separately. The chemical potential $\mu$ is obtained from the number equation. In each layer $j$, there is an "effective" chemical potential $V_{j}+\mu$ fixing the local density $\rho_{j}$. Based on these results for decoupled layers, the lowest-order perturbative expansion of the action with respect to the tunneling part $\left(t_{1}\right)$ yields

$$
\mathcal{S}_{\text {eff }, j \rightarrow j+1}^{\text {tunnel }}=-\int_{0}^{\hbar \beta} d \tau \int d \mathbf{x} T_{j \rightarrow j+1} \cos \left[\theta_{j+1}-\theta_{j}\right]
$$

with

$$
T_{j \rightarrow j+1}=\frac{t_{1}^{2} \rho_{j}}{2 \pi \hbar^{2} \rho_{j} / m+E_{b}},
$$

where $E_{b}$ is the binding energy of the molecule. This molecular binding energy can be derived from the scattering theory for the two-dimensional Fermi gas. It is given by [19]

$$
E_{b}^{2 \mathrm{D}}=0.583 \sqrt{s} E_{R} \exp \left(\frac{1}{\sqrt{2 \pi}} \frac{\lambda}{s^{-1 / 4} a_{s}}\right),
$$

where $a_{s}$ is the interatomic $s$-wave scattering length. It is important to note that the binding energy depends on the intensity and wavelength of the lasers generating the optical potential. More intense laser beams or smaller wavelengths confine the gas more strongly in the optical lattice and alter the binding energy of the resonant molecules. A more detailed determination of the molecular binding energy in an optical lattice, taking into account molecules formed from atoms in neighboring lattice sites, is given in Ref. [20].

The equations of motion for the remaining variables (density $\rho_{j}$ and phase $\theta_{j}$ in layer $j$ ) can be derived from the effective action (7)-(10) through the extremum conditions $\delta S_{\text {eff }} / \delta \theta_{j}=0$ and the number equation. This leads to the same equations as reported by the present authors and Wouters in Ref. [12]

$$
\begin{aligned}
\hbar \partial_{t} \frac{\rho_{j}(x)}{2}= & -\frac{\nabla \theta_{j} \nabla \rho_{j}}{4 m}+T_{j, j-1} \sin \left(\theta_{j}-\theta_{j-1}\right) \\
& -T_{j+1, j} \sin \left(\theta_{j+1}-\theta_{j}\right)
\end{aligned}
$$

and

$$
\begin{aligned}
-\hbar \partial_{t} \frac{\theta_{j}}{2}= & \frac{\left[\nabla \theta_{j}\right]^{2}}{8 m}+V_{j}-\mu-\frac{\partial T_{j+1, j}}{\partial \rho_{j}} \cos \left(\theta_{j+1}-\theta_{j}\right) \\
& -\frac{\partial T_{j, j-1}}{\partial \rho_{j}} \cos \left(\theta_{j}-\theta_{j-1}\right)
\end{aligned}
$$

Here, we investigate the case with a constant phase difference $\theta_{j+1}-\theta_{j}=\Delta \theta$ and a smoothly varying density $\rho_{j+1} \approx \rho_{j}$. This situation corresponds to a uniform flow of superfluid 
through the lattice. Such a situation can ideally only be achieved for an infinite lattice or in an optical lattice ring [21]. However, for large enough systems, boundary effects are negligible. Then Eqs. (13) and (14) simplify to

$$
\begin{gathered}
\hbar \partial_{t} \frac{\rho_{j}}{2}=\frac{t_{1}^{2}}{2 \pi \hbar^{2} \rho_{j} / m+E_{b}} \rho_{j} \sin (\Delta \theta) \\
-\hbar \partial_{t} \frac{\Delta \theta}{2}=\nabla_{j}\left(V_{j}\right)-\frac{t_{1}^{2} E_{b}}{\left[2 \pi \hbar^{2} \rho_{j} / m+E_{b}\right]^{2}} \cos (\Delta \theta) .
\end{gathered}
$$

In the BEC case, $E_{b} \gg \hbar^{2} \rho_{j} / m$ and we get the equations that describe a conventional Josephson junction array. However, on the BCS side, the tunneling coefficients start to depend on $\rho_{j}$, as $E_{b}$ and $\hbar^{2} \rho_{j} / m$ become comparable.

\section{CRITICAL JOSEPHSON CURRENT AND CRITICAL VELOCITY}

Equation (15) states that the current density $J$ is proportional to $\sin (\Delta \theta)$. This is similar to the first Josephson equation

$$
J=J_{c} \sin (\Delta \theta) .
$$

The second equation (16) fixes the time derivative of the phase difference to the differences in potential energy of the gas in the different wells. Near $\Delta \theta=0, \pi / 2$ Eq. (16) yields the second Josephson equation $-\hbar d(\Delta \theta) / d t=\Delta U$ where $\Delta U$ is the difference in energy between consecutive wells for the molecules. From the first Josephson equation we can extract a critical current by setting $\Delta \theta=\pi / 2$, as has been done for bosonic atoms in the optical lattice in Ref. [22]. This yields a critical current density for the Josephson tunneling from layer to layer

$$
J_{c}=\frac{2}{\hbar} \frac{t_{1}^{2}}{2 \pi \hbar^{2} \rho_{j} / m+E_{b}} \rho_{j} .
$$

The layers are separated by a distance $\lambda / 2$. From $J_{c}$ we can then extract the critical velocity for the fermionic atoms through the optical lattice

$$
v_{c}=\frac{t_{1}^{2}}{2 \pi \hbar^{2} \rho_{j} / m+E_{b}} \frac{\lambda}{\hbar} .
$$

The critical velocity of the fermionic superfluid depends on the scattering length $a_{s}$, via the binding energy of the Feshbach resonant molecule, $E_{b}$. The critical velocity also depends on the density (or, equivalently, the Fermi wave vector). In Fig. 1 we show the results for the critical velocity (expressed in microns per millisecond) as a function of $k_{F}$ and of the interaction parameter $1 /\left(k_{F} a_{s}\right)$. Here $k_{F}$ refers to the Fermi wave vector in the two-dimensional layer, so it is linked to the two-dimensional density of fermionic atoms in each layer and not to the overall three-dimensional density. In the region $1 /\left(k_{F} a_{s}\right)>0$ we are in the molecular BEC regime, and $E_{b} \gg \hbar^{2} \rho_{j} / m$. The critical velocity in the BEC regime is roughly proportional to $t_{1}^{2} / E_{b}$. In the region $1 /\left(k_{F} a_{s}\right)<0$, the BCS regime of Cooper pairs arises, and the

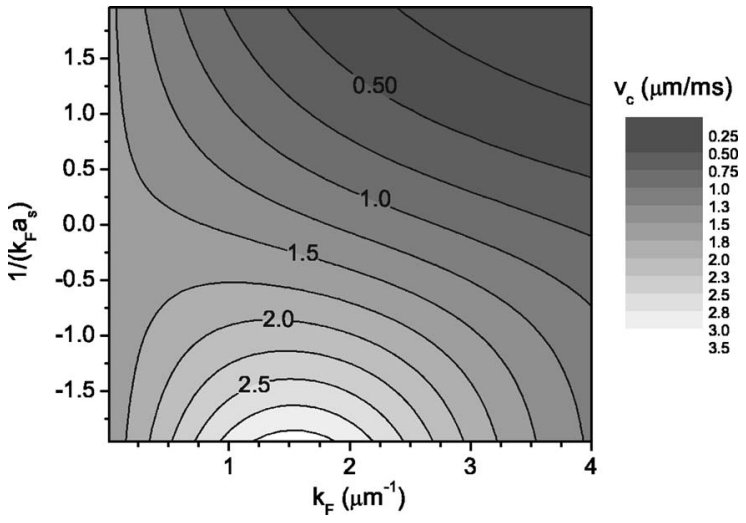

FIG. 1. The critical Josephson velocity through the optical lattice, in $\mu \mathrm{m} / \mathrm{ms}$, is shown as a function of the interaction strength parameter $1 /\left(k_{F} a_{s}\right)$ and the Fermi wave vector of the $2 \mathrm{D}$ fermi gas in the valleys. In the upper half $\left(a_{s}>0\right)$ of the figure the BEC regime develops, and in the lower half $\left(a_{s}<0\right)$ the BCS regime appears. The optical lattice parameters are $\lambda=795 \mathrm{~nm}$ and $s=0.5$. The atom parameters correspond to ${ }^{40} \mathrm{~K}$ atoms in the $|9 / 2,-7 / 2\rangle$ and $|9 / 2,-9 / 2\rangle$ hyperfine states, having a Feshbach resonance at $B=202.1 \mathrm{G}$.

result for the critical velocity becomes nontrivial. For each fixed value of $1 /\left(k_{F} a_{s}\right)<0$, there appears a maximum as a function of $k_{F}$. This maximum occurs when $E_{b} \approx \hbar^{2} \rho_{j} / m$, minimizing the denominator in (18). Figure 2 shows the partial derivative of this critical velocity with respect to the Fermi wave vector, at a constant value of the interaction parameter $1 /\left(k_{F} a_{s}\right)$. The maxima of the critical velocity as a function of $k_{F}$ are located on the contour $\partial v_{c} / \partial k_{F}=0$, shown as a thick curve in Fig. 2.

Although superfluid gases of bosonic atoms have already been studied in optical lattices [13,23], superfluid Fermi gases have not to this moment been loaded in optical lattices. Also no molecular condensates have been placed in optical lattices. For atomic condensates, a critical velocity could be

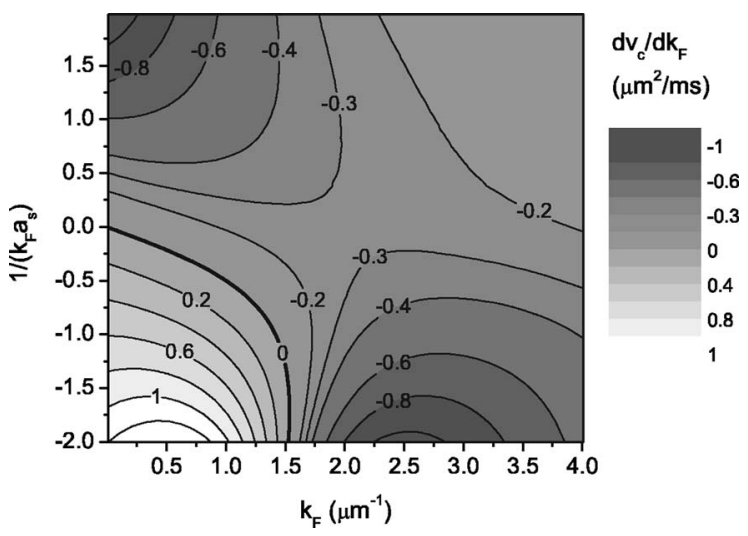

FIG. 2. The partial derivative of the critical velocity (in $\mu \mathrm{m}^{2} / \mathrm{ms}$ ) with respect to the Fermi wave vector, at constant value of the interaction parameter $1 /\left(k_{F} a_{s}\right)$, is shown as a function of the interaction parameter $1 /\left(k_{F} a_{s}\right)$ and the Fermi wave vector. The optical lattice parameters are $\lambda=795 \mathrm{~nm}$ and $s=0.5$. The atom parameters correspond to ${ }^{40} \mathrm{~K}$ atoms in the $|9 / 2,-7 / 2\rangle$ and $|9 / 2,-9 / 2\rangle$ hyperfine states, having a Feshbach resonance at $B=202.1 \mathrm{G}$. 
determined experimentally [23], and was found to vary between 0.2 and $1.2 \mu \mathrm{m} / \mathrm{ms}$ for ${ }^{87} \mathrm{Rb}$ atoms. This is comparable to the velocities that we predict for (fermionic) ${ }^{40} \mathrm{~K}$ in the same $\lambda=795$ optical potential. Thus, the superfluid regime of paired fermionic atoms in an optical lattice should be accessible experimentally. Note that for the wave numbers shown in Fig. 1, at $s=0.5$, the Fermi energy is smaller than the bandwidth of the Bloch band of the Fermi gas in the normal state, so that only the lowest Bloch band is occupied. When the laser intensity is increased, the Fermi wave vector that corresponds to the width of the Bloch band becomes smaller (for example, if $s=3$, then $E_{F} \approx \delta$ occurs at $k_{F} \approx 2 \mu \mathrm{m}^{-2}$ ).

\section{CONCLUSIONS}

The path-integral description of ultracold fermionic atoms interacting through a tunable contact potential allows to describe various non-ground-state configurations through a judicious choice of saddle point. We apply this formalism to the case of a fermionic gas in an optical potential. When the fermionic gas is in the superfluid regime, the layers of gas in the optical potential form a Josephson junction array. Equations of motion for the density and phase in each layer are obtained and applied to the case where the phase difference between consecutive layers is constant. This permits the derivation of a critical velocity of the superfluid flow through the optical potential. Although these results are strictly speaking derived for $T=0$, in the experiments the temperature can typically be brought down well below the degeneracy temperature so that we believe our results will be relevant to the experiments with optical lattices.

\section{ACKNOWLEDGMENTS}

Stimulating discussions with H. Kleinert and A. Pelster are gratefully acknowledged. J.T. acknowledges financial support from the FWO-Vlaanderen in the form of a mandaat "Postdoctoraal Onderzoeker van het FWO-Vlaanderen." This research has been supported financially by the FWO-V Projects Nos. G.0435.03, G.0306.00, the W.O.G. Project No. WO.025.99N, the GOA BOF UA 2000 UA, and the IUAP. J.T. gratefully acknowledges support of the Special Research Fund of the University of Antwerp, BOF NOI UA 2004.
[1] E. Tiesinga, B. J. Verhaar, and H. T. C. Stoof, Phys. Rev. A 47, 4114 (1993).

[2] M. W. J. Romans and H. T. C. Stoof, e-print cond-mat/ 0506282; G. M. Falco and H. T. C. Stoof, Phys. Rev. Lett. 92, 130401 (2004).

[3] G. B. Partridge, K. E. Strecker, R. I. Kamar, M. W. Jack, and R. G. Hulet, Phys. Rev. Lett. 95, 020404 (2005).

[4] M. W. Zwierlein et al., Phys. Rev. Lett. 91, 250401 (2003).

[5] K. M. O'Hara et al., Science 298, 2179 (2002).

[6] C. Menotti, P. Pedri, and S. Stringari, Phys. Rev. Lett. 89, 250402 (2002).

[7] C. A. Regal, M. Greiner, and D. S. Jin, Phys. Rev. Lett. 92, 040403 (2004).

[8] M. Bartenstein et al., Phys. Rev. Lett. 92, 120401 (2004).

[9] M. W. Zwierlein et al., Nature (London) 435, 1047 (2005).

[10] A. Bulgac and Y. Yu, J. Low Temp. Phys. 138, 741 (2005).

[11] J. Tempere, M. Wouters, and J. T. Devreese, Phys. Rev. A 71, 033631 (2005).

[12] M. Wouters, J. Tempere, and J. T. Devreese, Phys. Rev. A 70, 013616 (2004).
[13] F. S. Cataliotti et al., Science 293, 843 (2001).

[14] G. Orso, L. P. Pitaevskii, and S. Stringari, Phys. Rev. Lett. 93, 020404 (2004).

[15] C. A. R. Sá de Melo, M. Randeria, and J. R. Engelbrecht, Phys. Rev. Lett. 71, 3202 (1993).

[16] J. R. Engelbrecht, M. Randeria, and C. A. R. Sá de Melo, Phys. Rev. B 55, 15153 (1997).

[17] J.-P. Martikainen and H. T. C. Stoof, Phys. Rev. A 68, 013610 (2003).

[18] H. Kleinert, Fortschr. Phys. 26, 565 (1978); H. Kleinert, Ann. Phys. 266, 135 (1998).

[19] D. S. Petrov and G. V. Shlyapnikov, Phys. Rev. A 64, 012706 (2001).

[20] G. Orso, L. P. Pitaevskii, S. Stringari, and M. Wouters, e-print cond-mat/0503096.

[21] G.-P. Zheng and J.-Q. Liang, Commun. Theor. Phys. 43, 819 (2005).

[22] A. Smerzi, A. Trombettoni, P. G. Kevrekidis, and A. R. Bishop, Phys. Rev. Lett. 89, 170402 (2002).

[23] F. S. Cataliotti et al., New J. Phys. 5, 71 (2003). 